\title{
Development of a hollow fibre-based renal module for active transport studies
}

\author{
Alexandros Englezakis ${ }^{1}\left[\right.$ ] Elnaz Gozalpour ${ }^{2} \cdot$ Mohammed Kamran $^{1} \cdot$ Katherine Fenner $^{2} \cdot$ Elisa Mele $^{3}$. \\ Karen Coopman ${ }^{1}$
}

Received: 12 October 2020 / Accepted: 10 March 2021 / Published online: 22 March 2021

(c) The Author(s) 2021

\begin{abstract}
Understanding the active transport of substrates by the kidney in the renal proximal convoluted tubule is crucial for drug development and for studying kidney diseases. Currently, cell-based assays are applied for this this purpose, however, differences between assays and the body are common, indicating the importance of in vitro-in vivo discrepancies. Several studies have suggested that 3D cell cultures expose cells to a more physiological environments, thus, providing more accurate cell function results. To mimic the renal proximal tubule, we have developed a custom-made renal module (RM), containing a single polypropylene hollow fibre (Plasmaphan P1LX, 3M) that serves as a porous scaffold and compared to conventional Transwell cell-based bidirectional transport studies. In addition, a constant flow of media, exposed cells to a physiological shear stress of 0.2 dyne $/ \mathrm{cm}^{2}$. MDCK-Mdrla cells, overexpressing the rat Mdr1a (P-gp) transporter, were seeded onto the HF membrane surface coated with the basement membrane matrix Geltrex which facilitated cell adhesion and tight junction formation. Cells were then seeded into the HF lumen where attachment and tight junction formation were evaluated by fluorescence microscopy while epithelial barrier integrity under shear stress was shown to be achieved by day 7. qPCR results have shown significant changes in gene expression compared to cells grown on Transwells. Kidney injury marker such as KIM-1 and the hypoxia marker CA9 have been downregulated, while the CD133 (Prominin-1) microvilli marker has shown a fivefold upregulation. Furthermore, the renal transporter P-gp expression has been downregulated by 50\%. Finally, bidirectional assays have shown that cells grown in the RM were able to reabsorb albumin with a higher efficiency compared to Transwell cell cultures while efflux of the P-gp-specific substrates Hoechst and Rhodamine 123 was decreased. These results further support the effect of the microenvironment and fluidic shear stress on cell function and gene expression. This can serve as the basis for the development of a microphysiological renal model for drug transport studies.
\end{abstract}

Keywords Hollow fibre $\cdot$ 3D cell culture $\cdot$ Fluidic shear stress $\cdot$ Renal function $\cdot$ Drug transport

\section{Introduction}

Alexandros Englezakis

a.englezakis@lboro.ac.uk

1 Centre of Biological Engineering, Department of Chemical Engineering, Loughborough University, Loughborough, UK

2 Clinical Pharmacology and Safety Sciences, R\&D Biopharmaceuticals, AstraZeneca, Cambridge, UK

3 Department of Materials, Loughborough University, Loughborough, UK

A new drug is estimated to cost an average of $\$ 1.3$ billion to develop over a 12-year period with half of that time being used for pre-clinical in vitro testing [1]. It involves several stages, models and techniques, each providing data for the Administration, Distribution, Metabolism, Elimination, Toxicity (ADMET) properties of New Chemical Entities (NCE). This balance between stringent tests and increasing costs make drug development highly complex and requires the combined effort of a multidisciplinary team over a long duration to complete. Current methods of drug screening incorporate mainly computational models, recombinant systems and in vitro 2D cell-based assays to predict the 
ADMET properties of NCE before proceeding to clinical trials.

Although these methods have revolutionised and industrialised drug development in terms of efficiency and speed of NCE screening, $90 \%$ of new drugs fail during clinical trials [2]. This indicates the discrepancy between in vitro and in vivo drug testing, and with current high drug development costs and attrition rates during pre-clinical testing, the pharmaceutical industry faces increasing investment risks and decrease in return of investment while drug development time is increased. Therefore, it is essential to improve and develop new screening models to reduce both time and cost, while producing compounds with more favourable ADMET properties for clinical trials.

Based on US's Food and Drug Administration (FDA) regulatory guidelines, a new drug transport is suggested to study across the renal proximal tubule for better understanding the ADMET properties of a drug [3]. The renal convoluted proximal tubule is responsible for up to $80 \%$ of nutrient reabsorption/transport from the ultrafiltrate and drugs and their metabolites often accumulate in this region at high concentrations in intra- and intercellular spaces [4]. Thus, the proximal tubule is a major region of interest for studying the ADMET properties of NCEs and their interaction with important renal transporters belonging to the ATP-Binding Cassette (ABC) and Solute carrier (SLC) superfamilies [5-7].

A promising method for developing an accurate drugscreening model is the use of 3D renal cell cultures such as Organ-on-chips, organoids and bioartificial organs. Studies have shown that cells grown in a 3D environment using several methods, have characteristics closer to cells found in vivo, than their conventional 2D counterparts. These include cell growth, proliferation, phenotype, gene and protein expression profiles [8]. Unlike 2D cell cultures, cells grown in a 3D environment, provide a controllable environment in terms of $\mathrm{pH}$, temperature, nutrient supply, waste removal and shear stress [9]. However, limitations such as the even distribution of oxygen and nutrients make their use complex.

Therefore, the design of a 3D cell culture system is crucial for its function and these range from the selection of scaffold/matrix, which is then seeded with the desired cells, or dispersing cells in a liquid matrix followed by polymerization [8].

Aebischer et al. have developed a hollow fibre (HF)-based bioreactor to be used as a bioartificial kidney (BAK) device [10]. These semi-permeable HF membranes were made from either an acrylic polymer or polysulphone and were seeded on the outer surface with two types of renal epithelial cell lines (MDCK or LLC-PK1). They have shown that cell confluence on the hollow fibres was reached within 3 weeks and ultrafiltration was maintained continuously without any anticoagulation. However, the morphology and function of these cells was altered depending on the HF material and cell loss was observed in the long term [10].

This concept is now used widely for BAK development for both clinical and pharmacodynamics applications. Humes et al. developed this design further with the use of an extracellular protein coating (pronectin-L) to enhance cell attachment, growth and polarisation [11]. The same group has scaled up this system to a multifibre bioreactor with cells grown on the outer surface of the HF and the extracellular spaces serve as the proximal tubule lumen for pro-urine formation while the inner HF served as the peritubular capillary [12]. They tested the BAK device on uremic animals and have shown that these cells replaced several metabolic processes lost in renal failure that could not be replaced with conventional haemodialysis. These included glutathione metabolism, vitamin D production and sufficient ammonia excretion [12]. They have also tested the same BAK configuration on patients in ICU suffering from acute renal failure. Patients have shown some improvement in regard to cardiovascular stability and renal function as determined by urine production. In addition, there was an inflammatory cytokine reduction (IL-6 and IL-10), therefore, the BAK had a protective effect from systemic inflammatory response syndrome (SIRS) and multi-organ failure. However, most patients exhibited low platelet production and hypotension, resulting in the termination of the clinical study [13, 14].

Although these HF-based BAKs are being developed mainly for renal replacement therapies and have seen significant progress, they still lack some basic proximal tubule functions that take part in excretory, metabolic and endocrine pathways [9]. This is due to several reasons, but most important is the type of cell used (cell line or primary), materials used, scalability and bioreactor design $[15,17]$. In addition, the potential use of HF-based bioreactors for drug screening and renal transport studies has not been fully explored.

In this study, we have sought to develop a HF-based renal module (RM) similar to BAKs to be used as an in vitro renal transport model. A custom-made bioreactor was manufactured that houses a single Polypropylene HF (Plasmaphan P1LX, 3M).

It was hypothesised that due to their porosity and curvature, HFs are an ideal scaffold for renal proximal tubule epithelial cells, as it mimics the proximal convoluted tubule in the nephron, therefore, providing a similar 3D microenvironment. In addition, cells would be exposed to fluidic shear stress on both sides of the HF membrane to mimic the ultrafiltrate flow in the proximal tubule and blood flow in the peritubular capillaries. Renal MDCKMdrla cells were seeded in the HF lumen, while syringe 
pumps provided a constant flow of media through the inner and outer HF membrane surface. Cells were then evaluated according to their tight cell monolayer formation, gene expression levels and renal transporter functionality compared to cells grown on Transwell inserts.

\section{Materials and methods}

\section{Chemicals}

Human collagen IV $(0.3 \mathrm{mg} / \mathrm{ml})$ and Valspodar were purchased from Sigma-Aldrich. DMEM (4.5 g/l glucose with L-glutamine, sodium pyruvate), Penicillin-Streptomycin (P/S, $100 \mathrm{U} / \mathrm{ml}$ ), Foetal Bovine Serum (FBS), Fibronectin (from bovine plasma), rhLaminin521 (1 mg), Geltrex hESCQualified (Reduced Growth Factor Basement Membrane Matrix), Gibco TryplE, Rhodamine123, Hoechst 33,342, PBS $\left(\mathrm{CaCl}_{2}, \mathrm{MgCl}_{2}\right.$ free, PFA (4\%), anti-ZO-1 AF488 monoclonal antibody $(1 \mathrm{mg} / \mathrm{ml})$, AF-555 anti-rabbit antibody, BSA-AF594 and TRIzol were purchased from ThermoFisher Scientific. Plasmaphan P1LX HFs $(0.45-\mu \mathrm{m}$ pore size, $330-$ $\mu \mathrm{m}$ OD, $150-\mu \mathrm{m}$ ID) were purchased by $3 \mathrm{M}$. MDCK-Mdr1a cell line overexpressing the rat P-gp was supplied by AstraZeneca. Anti-rat P-gp rabbit antibody was purchased from Abcam.

\section{Cell cultures}

MDCK-Mdr1a cells were grown in DMEM (4.5 g/l glucose with L-glutamine, sodium pyruvate) media supplemented with $10 \% \mathrm{FBS}$ and $\mathrm{P} / \mathrm{S}(1 \mathrm{U} / \mathrm{ml})$. Cells were expanded in conventional tissue culture plastic (TCP) and were always passaged when cells reached a confluency of $80 \%$.

\section{HF coating and cell seeding on outer surface}

P1LX HFs were cut into 3-cm segments, placed in 6-well ultra-low attachment plates, and held down to the bottom by stainless steel rings. A mixture of Ethanol/deionised water (70/30) was used to sterilise the HFs, followed by three washes with sterile water. Sterile HFs were then coated with either Human Collagen IV $(200 \mu \mathrm{g} / \mathrm{ml})$, rhLaminin521 $(10 \mu \mathrm{g} / \mathrm{ml})$, Fibronectin $(10 \mu \mathrm{g} / \mathrm{ml})$ or Geltrex $(150 \mu \mathrm{g} / \mathrm{ml})$ by submerging the HFs in the solutions and incubated $1 \mathrm{~h}$ at $37^{\circ} \mathrm{C}$. After coating, $4 \times 10^{5}$ MDCK-Mdr1a cells were seeded and incubated for 3 days.

\section{SEM and fluorescence microscopy imaging}

For SEM images, cells were fixed with 4\% PFA (w/v), and after washing with PBS, they were left to dry at room temperature for at least 3 days. SEM images were taken using a Timochi, Benchtop SEM microscope (TM4000) and representative images are shown.

For fluorescence imaging after fixation, cells were stained with anti ZO-1 AF488 $(2.5 \mu \mathrm{g} / \mathrm{ml})$ conjugated antibody with or without anti P-gp $(7 \mu \mathrm{g} / \mathrm{ml})$ primary antibody overnight at $4{ }^{\circ} \mathrm{C}$ following with a 10 -min DAPI $(0.1 \mu \mathrm{g} /$ ml) staining the next day. Samples with anti P-gp antibody were stained with AF-555 anti-rabbit antibody for 4 $\mathrm{h}$ at room temperature as well. After washing with PBS, samples were visualised with a Nikon, Eclipse Ti2 series fluorescent microscope.

For images showing the inner surface, cells were fixed and stained by injecting the prepared solution using a syringe inside the HF lumen. After staining and washing, the HFs were cut open diagonally or vertically with a scalpel to expose the inner surface.

\section{Renal module design and assembly}

Four identical modules were designed to house a single 5-cm-long Plasmaphan P1LX HF with an extra capillary space (ECS) with a volume capacity of $0.25 \mathrm{ml}$ while Luer lock stainless needles ( $30 \mathrm{G}$ ) were fed into the HF lumen (L). Independent inputs and outputs for both ECS and L were introduced into the modules and four-way stopcocks with male and female Luer locks were used to allow for module bypass to the waste bottle for air bubble removal and quick media change. Once assembled, the modules were connected to 10-ml BD Luer lock syringes while the flow was controlled by a syringe pump (Harvard PHD ULTRA ${ }^{\mathrm{TM}}$ ). PTFE tubing (Eppendorf DASGIP feed line) was used for connecting inputs/outputs from the feeding syringes to the collecting/waste bottles. Details on the BAK design and set up are shown in Fig. 1, Supporting information.

\section{Cell seeding}

Once assembled, the modules were sterilised with $10 \mathrm{ml}$ of $70 \%$ Ethanol with a flow rate of $100 \mu \mathrm{l} / \mathrm{min}$, followed by a PBS wash of same volume and flow rate. HFs inside the modules were coated by injecting $1 \mathrm{ml}$ of Geltrex in the lumen and incubate for $1 \mathrm{~h}$ at $37^{\circ} \mathrm{C}$. After coating, $1 \mathrm{ml}$ of MDCK-Mdr1a cells $\left(2 \times 10^{6} / \mathrm{ml}\right)$ was injected into the lumen while the ECS was filled with DMEM complete cell culture media. After $24 \mathrm{~h}$, unbound cells were flushed by applying a flow rate of $100 \mu \mathrm{l} / \mathrm{min}$ for $5 \mathrm{~min}$. Afterwards, a shear stress 
of $0.2 \mathrm{dyne} / \mathrm{cm}^{2}$ was applied by perfusing media with a flow rate of $3 \mu \mathrm{l} / \mathrm{min}$ in both $\mathrm{L}$ and ECS. Cells grown in static conditions were seeded as mentioned above with daily media changes by applying a flow of $50 \mu \mathrm{l} / \mathrm{min}$ for $5 \mathrm{~min}$. The flow rate was calculated using the following equation:

$t=4 \mu Q / \pi R^{3}$,

where $t$ is shear stress (dyne. $\left.\mathrm{cm}^{2}\right), \mu$ is media viscosity (Cp), $Q$ is flow rate $\left(\mathrm{cm}^{3} / \mathrm{s}\right)$ and $R$ is HF radius (cm).

\section{Cellular monolayer integrity assessment}

To assess the tightness of monolayer in Transwell cell cultures, separate inserts were treated with Inulin-FITC $(50 \mu \mathrm{g} / \mathrm{ml})$ on the apical side and only plates with a $P_{\text {app }}<1 \times 10^{-6} \mathrm{~cm} / \mathrm{s}$ were considered to perform efflux assay.

In $3 \mathrm{D}$ cell culture, media flowing though the HF Lumen contained Inulin-FITC $(50 \mu \mathrm{g} / \mathrm{ml})$ and cell confluency was assessed daily by collecting sample from the ECS and measuring media fluorescence using a FLUOstar Omega plate reader at $485 \mathrm{~nm}$ ex-520 $\mathrm{nm}$ em. Once Inulin-FITC diffusion decreased and was stable for at least 3 days, it was assumed that cell confluency and monolayer formation was achieved in the HF lumen.

\section{qPCR}

Cells grown under shear stress conditions were harvested and lysed in TRIzol. Total RNA was isolated, using the RNease Mini kit (Qiagen) followed by cDNA synthesis by HighCapacity RNA-to-cDNA ${ }^{\mathrm{TM}}$ kit (FisherScientific). Primers were designed and optimised for their specific targets (Table 1, Supporting Information). RT-PCR was performed with SYBR green master mix (ThermoFisher Scientific) and GAPDH was used as housekeeping gene, and relative expression levels were calculated as fold change from gene expression levels in 2D static cell culture (Transwells) using the $2^{-\Delta \Delta \mathrm{CT}}$ method. Statistically significant differences were determined by Student's $t$ test, where a $p$ value of less than 0.05 was considered statistically significant.

\section{Lactate and glucose consumption}

For Transwell cell cultures, $1 \times 10^{5}$ MDCK-Mdr 1a cells were seeded in a 24-well plate and daily samples were collected.

For RM cell cultures, cells were cultured as described above under flow conditions and samples were collected from the Lumen side of each module daily. Lactate and Glucose concentrations were measured using a Cedex Bio Analyzer (Roche Cedex Analyzer).

\section{Bidirectional transport assays}

To study conventional Transwell cell culture bidirectional transport assays, $0.1 \times 10^{6}$ MDCK-Mdr1a cells were seeded onto Corning Transwell inserts (12-well plate) and cultured for 10 days. The assessment of cellular monolayer and efflux assays of both Transwell and RM cell cultures were performed in parallel. The Transwell upper chamber is the Apical side while the lower chamber is the Basolateral side. The RMs HF Lumen (L) and Extracapillary space (ECS) were considered as the corresponding Apical and Basolateral sides, respectively.

Direction of substrate transport from the donor to the recipient side is shown with the symbols $\mathrm{A}>\mathrm{B}$ (Apical to Basolateral) and B > A (Basolateral to Apical).

For Transwell BSA transport studies, after 10 days of culture, BSA-AF594 $(50 \mu \mathrm{g} / \mathrm{ml})$ diluted in DMEM media was added either in the Apical or Basolateral side of Transwell inserts and samples were collected from the receiver sides every hour.

For RM BSA transport, BSA-AF594 $(50 \mu \mathrm{g} / \mathrm{ml})$ diluted in DMEM was included in either the ECS $(\mathrm{B}>\mathrm{A})$ media or Lumen $(B>A)$ media. Using a continuous flow rate of $3 \mu \mathrm{l} / \mathrm{min}$, samples from the receiver sides were collected every hour.

For Transwell P-gp active transport assays, cells were grown for 10 days on Transwell inserts. On the final day, the inserts were washed with fresh media and incubated with $0.5 \mu \mathrm{M}$ Valspodar or corresponding amount of DMSO for positive controls for $30 \mathrm{~min}$. After that, media was prepared containing $20 \mu \mathrm{M}$ Hoechst 33342 or $10 \mu \mathrm{M}$ Rhodamine 123 with/without Valspodar $(0.5 \mu \mathrm{M})$. Apical to Basolateral $(\mathrm{A}>\mathrm{B})$ transport was measured by adding the fluorescent substrate in the apical side and taking samples every $30 \mathrm{~min}$ from the basolateral side. For Basolateral to Apical $(\mathrm{B}>\mathrm{A})$ transport, the substrate was added on the basolateral side, and samples from the apical side were taken.

For RM P-gp active transport assays, MDCK-Mdr1a cells were seeded in the RM as described above. On day 9, cells were treated for $24 \mathrm{~h}$ with complete media containing the inhibitor Valspodar $(0.5 \mu \mathrm{M})$ or equivalent amounts of DMSO. Afterwards, the ECS and Lumen sides were flushed completely and replaced with either DMEM with/ without Valspodar $(0.5 \mu \mathrm{M})$ or DMEM with/without Valspodar and fluorescent substrate $(20 \mu \mathrm{M}$ Hoechst or $10 \mu \mathrm{M}$ Rhodamine 123). Media perfusion was applied $(3 \mu \mathrm{l} / \mathrm{ml})$ and samples were collected every hour from the receiver sides.

Apparent permeability coefficients $\left(P_{\text {app }}\right)$ were calculated from the following equation: 
$P_{\text {app }}=\frac{Q / t}{V\left(C_{0} A\right)}$,

where $Q$ is sample fluorescence (RFU), $d t$ is time (s), $V$ is receiver side volume (ml), $C_{0}$ is donor fluorescence intensity (RFU) and $A$ is insert membrane surface area $\left(\mathrm{cm}^{2}\right) . P_{\text {app }}$ ratios were calculated by calculating the ratio between $P_{\text {app }}$ (B $>$ A) and $P_{\text {app }}(\mathrm{A}>\mathrm{B})$. For cellular monolayer assessment, separate inserts were treated with Inulin-FITC $(50 \mu \mathrm{g} / \mathrm{ml})$ on the apical side and only plates with a $P_{\text {app }}<1 \times 10^{-6} \mathrm{~cm} / \mathrm{s}$ were considered. Hoechst fluorescence was measured at $355 \mathrm{~nm}$ ex/520 nm em while Rhodamine123 was measured at $485 \mathrm{~nm}$ ex/520 nm em.

Statistically significant differences were determined by Student's $t$ test, where a $p$ value of less than 0.05 was considered statistically significant.

\section{Results}

\section{HF coating}

First, a coating with a suitable basement membrane matrix protein was examined. As the aim of this initial study was for cells to attach in sufficient numbers on the HF membrane, cells were seeded on outer surface only. Comparison of MDCK-Mdr1a attachment on coated P1LX HFs using different basement matrix proteins is shown in Fig. 1. Cell adhesion and HF coverage is shown to be greatly affected on the type of basement matrix protein used. Greater cell HF coverage is seen by HFs coated with Geltrex, Laminin and Fibronectin. Limited cell coverage is seen when human collagen IV coating was used and almost no cells were seen on uncoated HFs. Fluorescent staining of P1LX HFs has shown greater cell densities on Geltrex-coated HFs (Fig. 2). Furthermore, ZO-1 staining indicates tight junction formation on cells attached on HFs.

\section{Effect of shear stress on MDCK-Mdr1a cells}

We next sought to examine whether MDCK-Mdr1a cells can withstand constant shear stress due to media perfusion though the RM. Cells were seeded in the HF lumen of the RM and were exposed to a constant flow of media after $24 \mathrm{~h}$ to induce shear stress. Figure 3 shows SEM images of open P1LX HFs with cells grown in static or flow conditions. Cells grown in static conditions show gaps and inconsistent cell monolayer (Fig. 3b). In contrast, cells exposed to shear stress seem have complete HF surface coverage with minimal gaps (Fig. 3c).

This is further supported by fluorescence microscopy images that show MDCK-Mdr1a cells cultured in flow conditions, grew in higher densities, and formed clear tight junctions due to staining of ZO-1 marker compared to cells grown in static conditions (Fig. 4). P-gp expression is seen in both conditions (Fig. 4c, g). Furthermore, HF crosssections show differences in cell growth between the two conditions. Statically grown cells form clusters towards
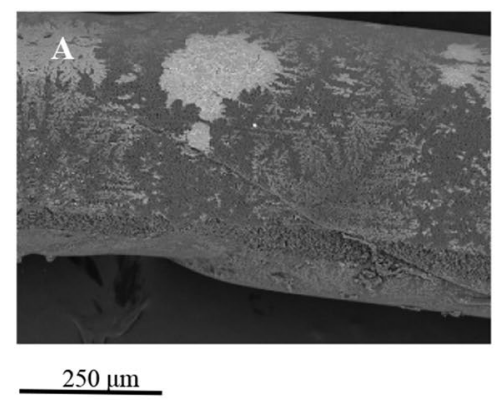

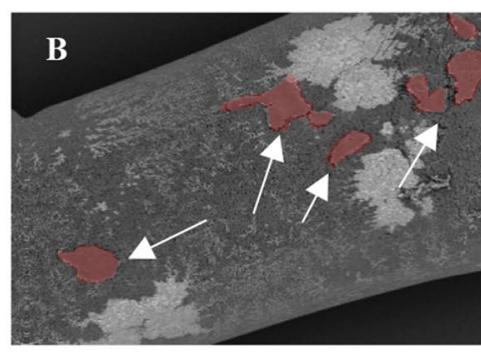

$250 \mu \mathrm{m}$

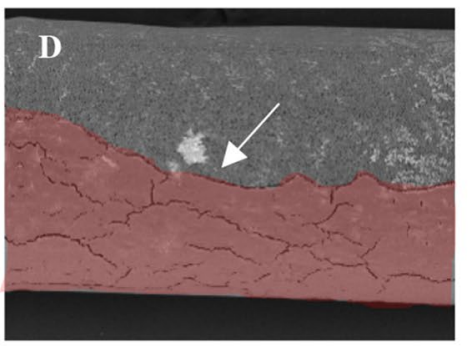

$250 \mu \mathrm{m}$

Fig. 1 Representative SEM images of P1LX HFs seeded with MDCK-Mdr1a cells for $72 \mathrm{~h}$ on their outer surface. HFs were either uncoated (a) or coated with $200 \mu \mathrm{g} / \mathrm{ml}$ Human Collagen IV (b),

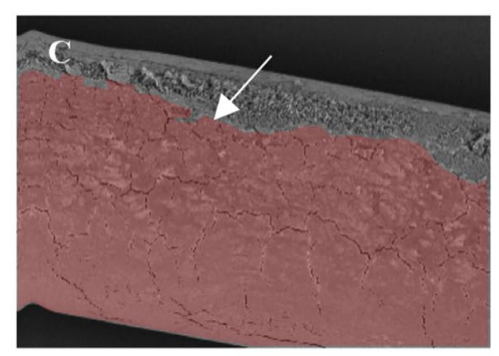

$250 \mu \mathrm{m}$

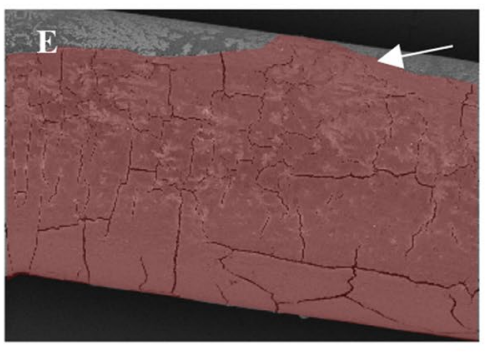

$250 \mu \mathrm{m}$

$10 \mu \mathrm{g} / \mathrm{ml} \mathrm{rhLaminin} 521$ (c), $10 \mu \mathrm{g} / \mathrm{ml}$ Fibronectin (d) or $150 \mu \mathrm{g} / \mathrm{ml}$ Geltrex (e). Cells are highlighted in red and shown by arrows 

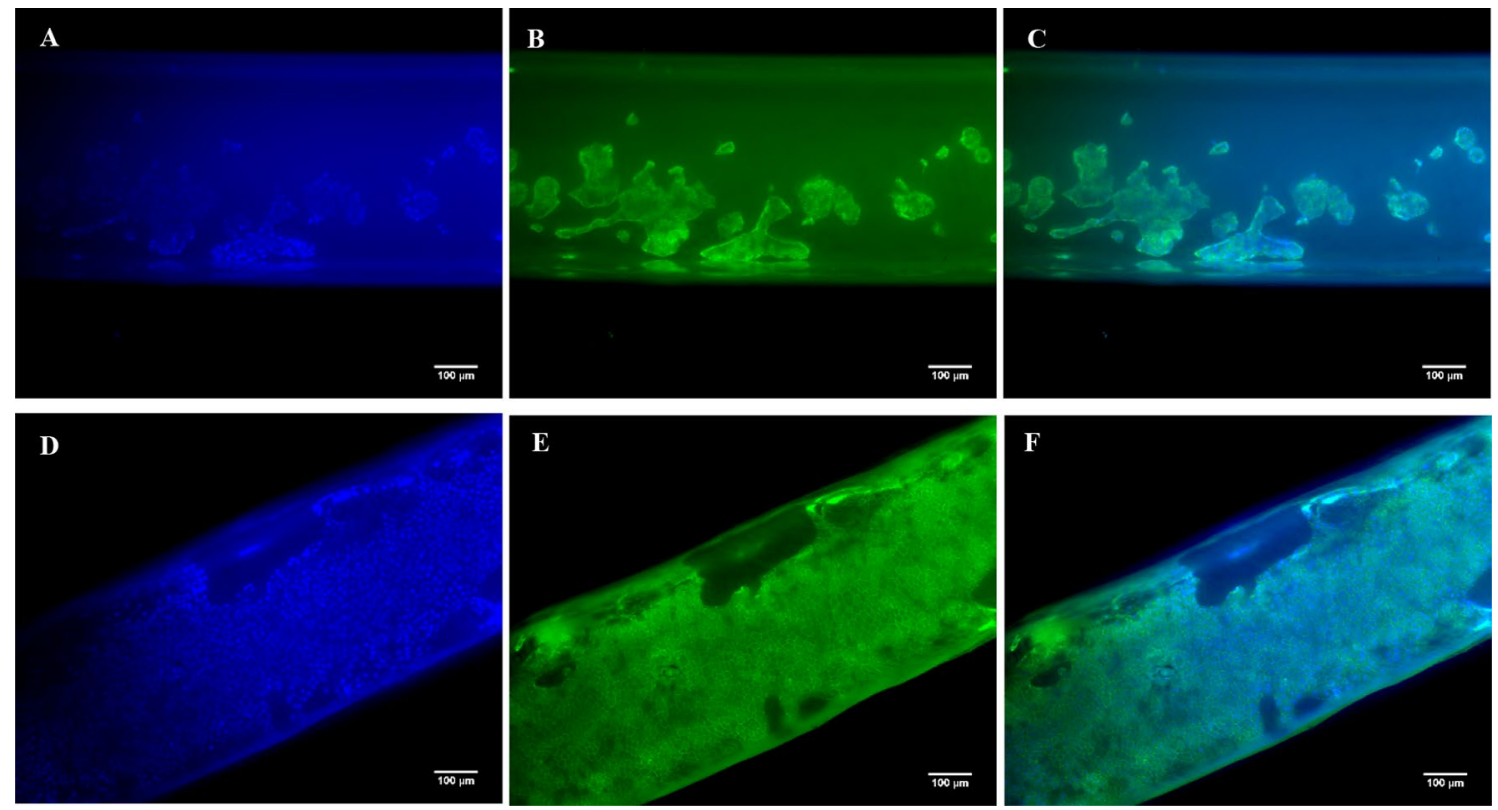

Fig. 2 Representative fluorescent microscopy images of MDCK-Mdr1a seeded for $72 \mathrm{~h}$ on the outer surface of uncoated (a-c) and coated (d-f) P1LX HF membrane. Cells are shown stained with DAPI (a, d), anti ZO-1 AF488 (b, e). Merge of both channels are also shown (c, f)

Fig. 3 Representative SEM images of $\mathrm{HF}$ inner surfaces without cells (a) and MDCKMdr1a cells, cultured in the renal module under static (b) and shear stress conditions (c)

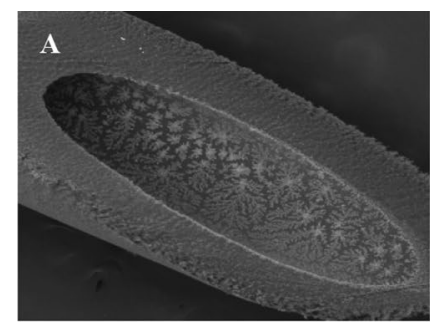

$250 \mu \mathrm{m}$

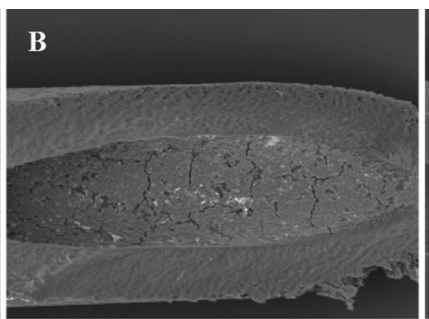

$250 \mu \mathrm{m}$

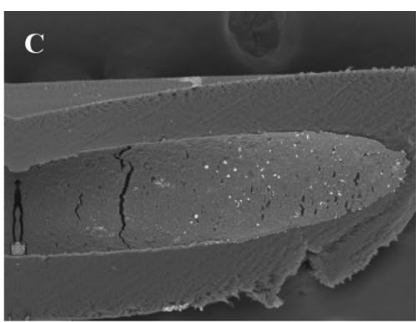

$250 \mu \mathrm{m}$
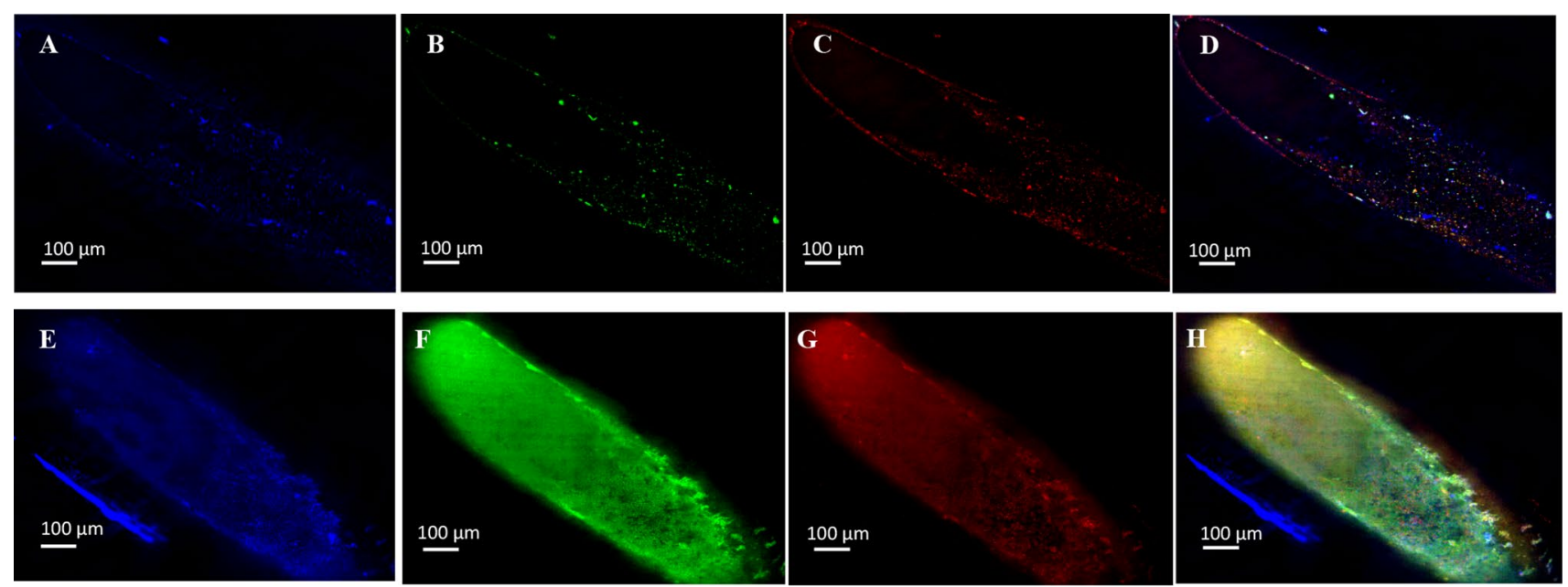

Fig. 4 Representative fluorescent microscope images of HF inner surfaces with MDCK-Mdrla cells grown in coated HFs under static (a-d) and fluid shear stress (e-i) conditions. Cell nuclei were stained

with DAPI (a, e), and ZO-1 (b, f) and P-gp (c, g) were stained with their corresponding antibodies and channels were merged $(\mathbf{d}, \mathbf{g})$ 

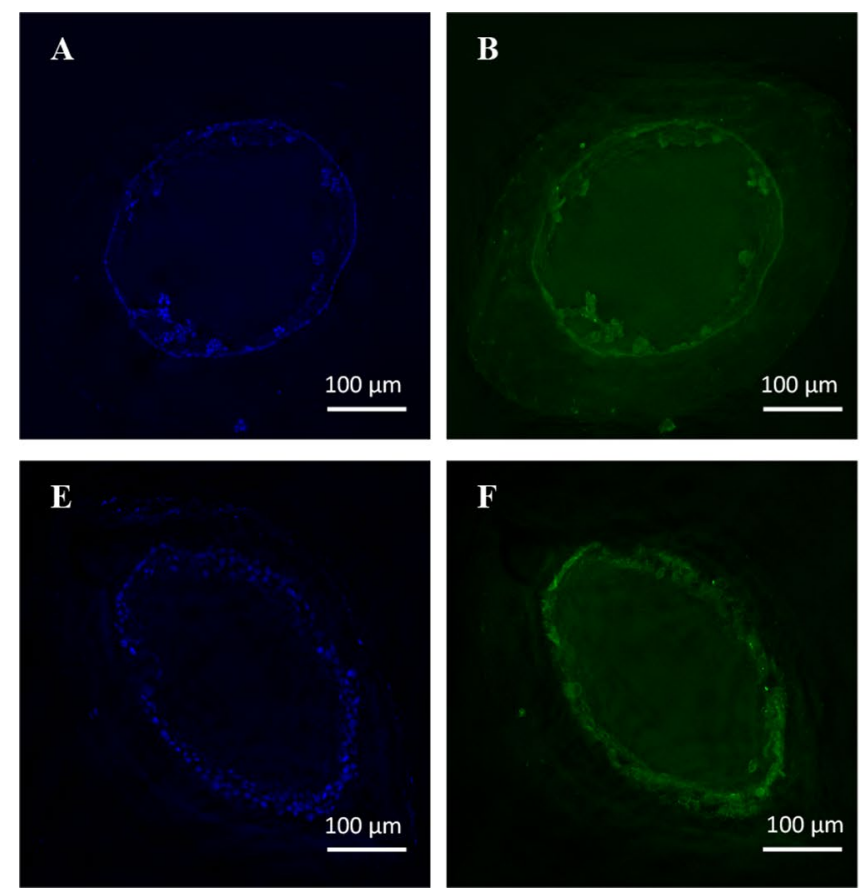

Fig. 5 Representative fluorescent microscope cross-section images of HFs seeded with MDCK-Mdrla cells grown under static (a-d) and fluid shear stress (e-i) conditions. Cell nuclei were stained with DAPI

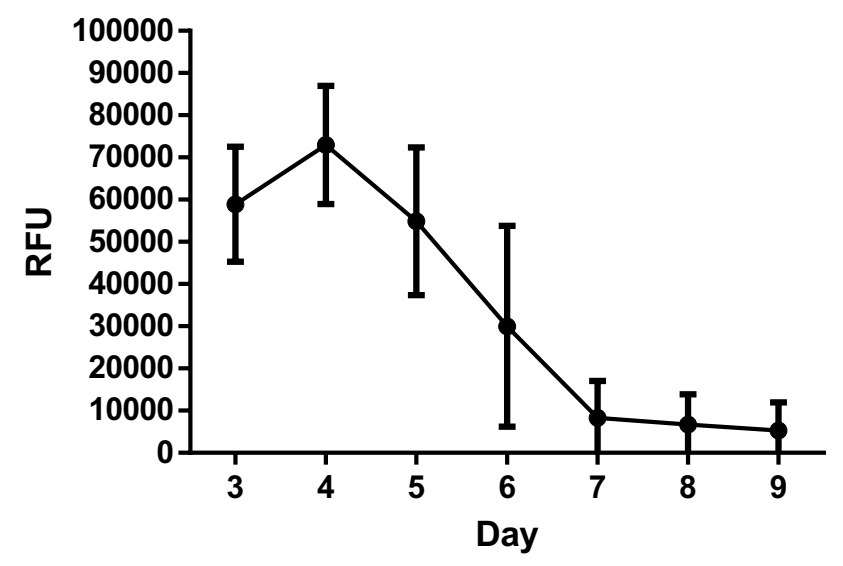

Fig. 6 Daily diffusion of Inulin-FITC from HF lumen to the outer ECS chamber in the RM (mean value $\pm \mathrm{SD}, n=3$ )

the lumen centre, while shear stress induces the formation of a cell monolayer on the HF inner surface (Fig. 5).

\section{Cellular monolayer integrity in renal modules}

Cell confluency and tight junction formation were assessed by the diffusion of the fluorescent marker Inulin-FITC from the HF lumen to the ECS. Inulin-FITC diffusion to the ECS is decreased and reached minimal levels by day 7
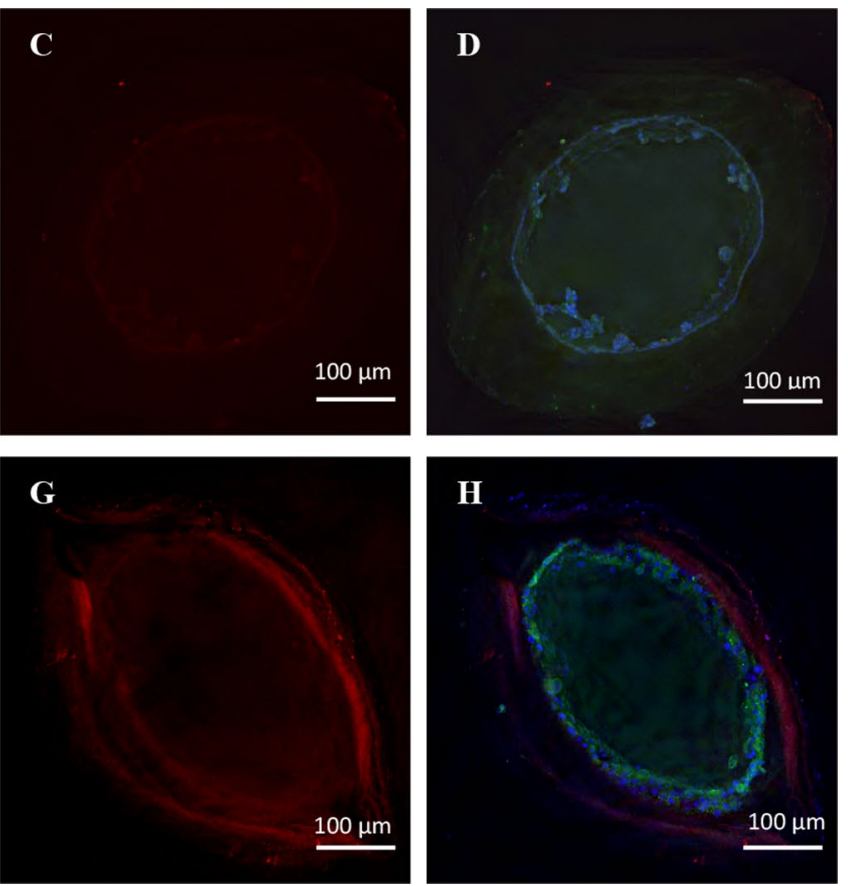

(a, e), and ZO-1 (b, f) and P-gp (c, g) were stained with their corresponding antibodies and channels were merged $(\mathbf{d}, \mathbf{g})$

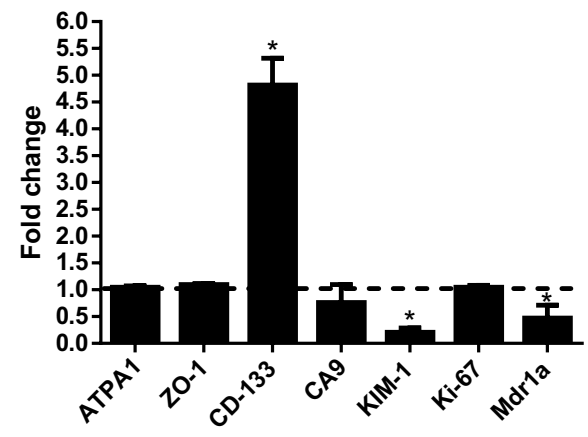

Fig. 7 Gene expression fold change in MDCK-Mdr1a cells seeded in the RMs under shear stress. Gene expression levels were normalised to GAPDH housekeeping gene and fold change was calculated to gene expression levels of cells grown on Transwell inserts. Dotted line indicates the normalised value of " 1 " for $2 \mathrm{D}$ cell cultures' gene expression profile. $* p<0.05$

and maintained this until day 9 indicating the presence of an intact cellular barrier (Fig. 6).

\section{qPCR}

Later, we studied the changes in gene expression between polarised MDCK-Mdr1a cells grown on Transwells and cells grown in the RMs under flow conditions (Fig. 7). CD133 (Prominin-1) has shown a fivefold increase compared to 
Fig. 8 Representative data of daily glucose concentration (a) and lactate concentration (b) of cells grown in the RM under flow conditions and compared to static Transwell cell cultures (mean value $\pm \mathrm{SD}, n=2$ )
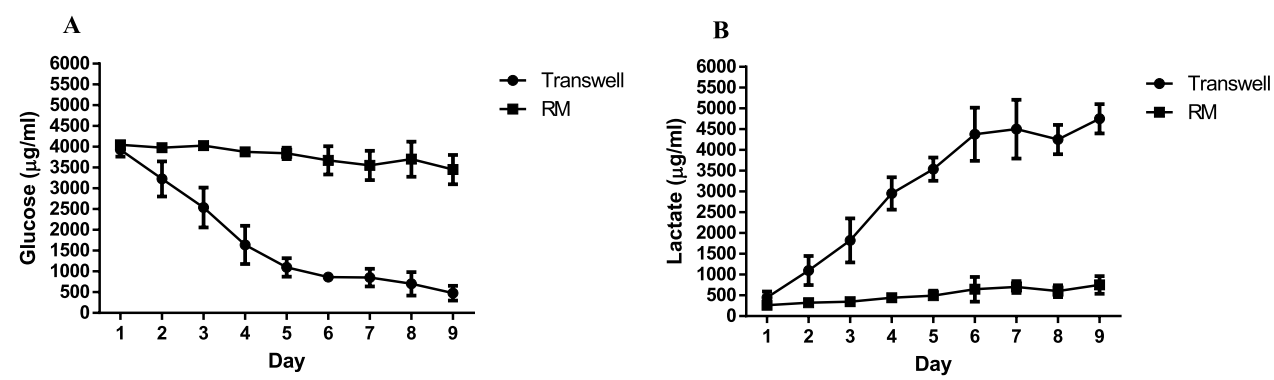

cells grown in 2D, while the kidney injury molecule KIM-1 and the renal transporter P-gp (Mdr1a) have shown a downregulation of $80 \%$ and $50 \%$, respectively. $\mathrm{Na}^{+} / \mathrm{K}^{+}$ATPase (ATPA1), tight junction protein (ZO-1), carbonic anhydrase 9 (CA9) and proliferation marker (Ki-67) have shown no significant changes.

\section{Glucose and lactate consumption (Transwell vs RM)}

Glucose consumption and Lactate accumulation were measured daily in MDCK-Mdr la cell cultures grown in the RMs and compared to conventional Transwell cell cultures (Fig. 8). Transwell cell cultures have shown a steady decrease and increase of Glucose and Lactate, respectively, while RM cell cultures maintained constant concentrations under flow conditions.

\section{Bidirectional transport assays (Transwell vs RM)}

The ability of cells grown in a 3D environment in the RMs under flow conditions to transport specific substrates was studied and compared to static Transwell cell cultures. BSA reabsorption and transport from the apical to the basolateral sides was shown to increase steadily (Fig. 9a, b) in both conditions, while permeability coefficient ratios of 3D cell cultures have shown a 20-fold increase in BSA reabsorption compared to 2D Transwell cell culture models (Fig. 9c).

We next sought to study the function of the apical transporter P-gp and compare its Hoechst and Rho123 transport activity in Transwell and RM cell cultures. Clear efflux of both Hoechst and Rho123 from the basolateral side (ECS) to the apical side $(\mathrm{L})$ is seen in the RMs while inhibition with Valspodar confirms the P-gp mediated transport (Fig. 10b, e). Comparison with Transwell cell culture assays show a reduction of $P_{\text {app }}$ ratios in RMs, indicating a decrease in P-gp function. Significant reduction is seen in Hoechst efflux, while Rho123 transport was affected slightly compared to Transwell-based assays (Fig. 10c, f).

\section{Discussion}

In this study, we sought to develop a HF-based RM to be used as a more physiological model for bidirectional transport. The HF of choice was Plasmaphan P1LX, 3M. This polypropylene-based HF membrane is inherently hydrophobic, and thus, unfavourable for cell adhesion. However, Buschmann et al. have managed to develop an artificial artery model by seeding endothelial cells on fibronectincoated P1LX HFs suggesting the necessity of basement membrane proteins in supporting cell attachment [18].
A

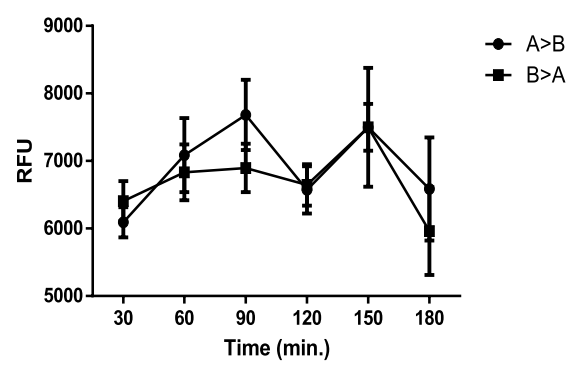

B

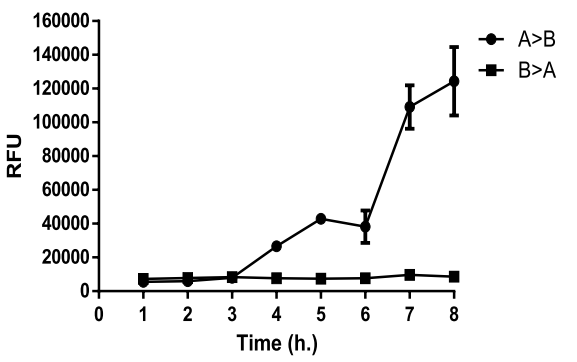

C

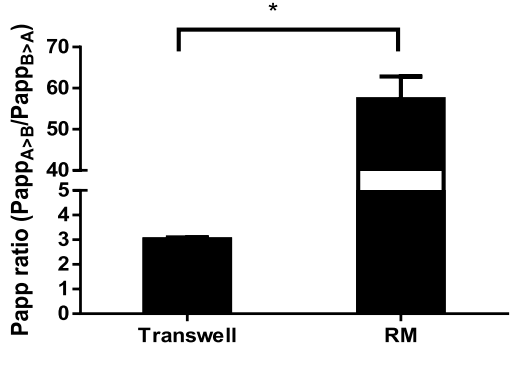

Fig. 9 Representative data of BSA-AF594 transport in MDCK-Mdr1a cells grown on Transwell inserts (a) and in RM cell cultures (b). Permeability coefficient $\left(P_{\text {app }}\right)$ ratios were calculated from 2 separate experiments, $p$ values calculated using a paired $t$ test were $* p<0.05$ (c) (mean value $\pm \mathrm{SD}, n=2$ ) 
A

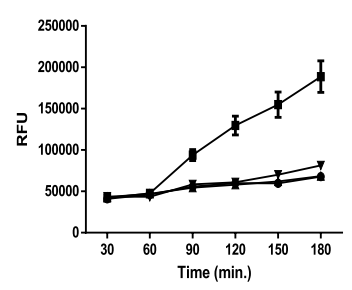

D

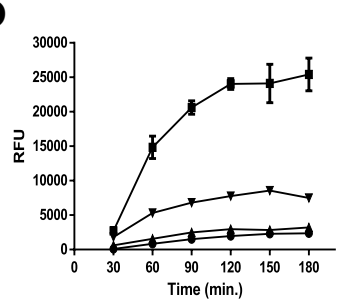

B
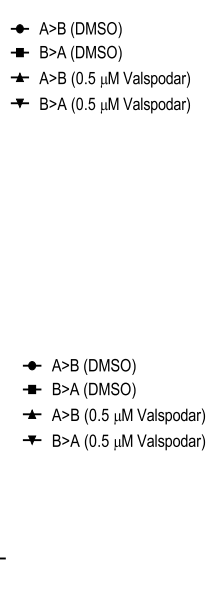

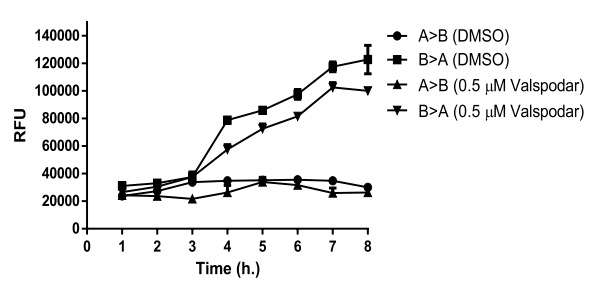

$\mathbf{E}$

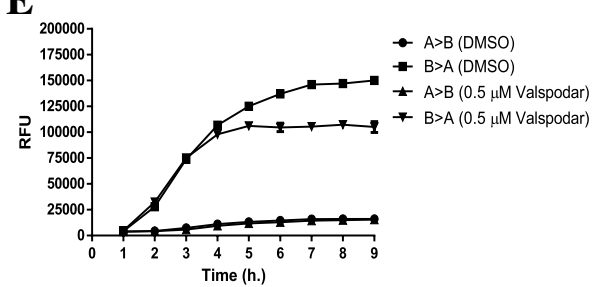

C

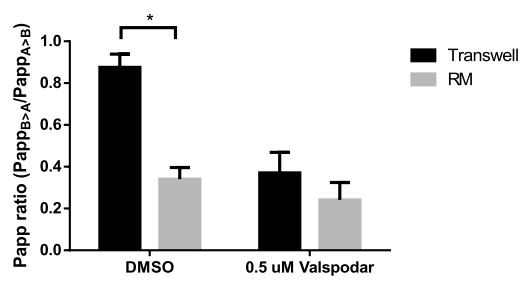

F

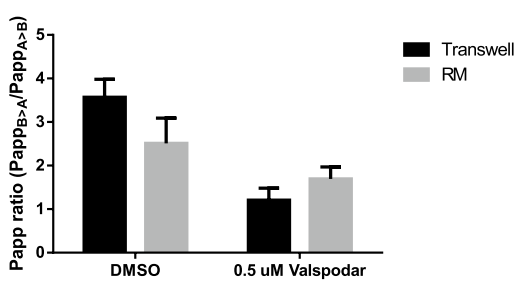

Fig. 10 Representative data of efflux of the P-gp-specific substrates Hoechst $(\mathbf{a}-\mathbf{c})$ and Rho123 (d-f) and a comparison between Transwell efflux (a, d) and RM efflux (b, e). Permeability coefficient
$\left(P_{\text {app }}\right)$ ratio comparison between the two models are also shown $(\mathbf{c}, \mathbf{f})$ and statistical significance was calculated using a paired $t$ test where ${ }^{*} p<0.05$ (mean value $\pm \mathrm{SD}, n=3$ )

proximal tubule epithelial cells are under constant fluid shear stress and are able to adapt to blood and ultrafiltration flow fluctuations [33]. Furthermore, 3D renal models with other cell lines and bioreactors have suggested a connection between shear stress and renal transport gene expression $[34,35]$. The exact mechanism that causes this is still unknown.

We have shown that when exposed to shear stress, cells were grown in greater numbers and have had an almost complete coverage of the HF lumen. In addition, when exposed to shear stress, MDCK-Mdrla cells formed tight junctions and a monolayer while under static conditions, cells formed clusters growing towards the HF lumen centre, while staining of the ZO-1 marker decreased. P-gp staining has shown expression of Mdr1a in cells grown in either static or flow conditions.

For qPCR studies, we have examined seven markers that are involved in renal cell function. ATPA1 expresses for $\mathrm{Na}^{+} / \mathrm{K}^{+}$ATPase, an important ion pump found in a wide variety of cell types including kidney epithelial cells and play an important role in regulating signal transduction pathways [36]. ZO-1 is highly expressed in cells forming tight junctions while CD133 is present in cells that have plasma membrane protrusions such as microvilli [37, 38]. CA9 is a widely used endogenous marker for hypoxic cells and KIM-1 is a kidney injury marker expressed in epithelial cells under stress or epithelial cell damage [39, 40]. Finally, proliferation was observed by measuring Ki-67 expression and P-gp function by Mdr1a expression.

qPCR results have shown a fivefold upregulation of the microvilli marker CD133 in comparison to static Transwell cell cultures. This suggests an increase in cell membrane phology microvilli formation and function [30-32]. Renal 
protrusion activity, and it may involve increased microvilli length and function. Furthermore, KIM-1, a marker of acute kidney injury, is downregulated by $\sim 80 \%$, indicating that cells are not under stress or damaged [40]. In addition, CA9 was slightly downregulated, meaning cells are not under hypoxic conditions in the RM. Furthermore, ATPA1 and Ki-67 have remained unchanged, indicating cells have a comparable metabolism and proliferation rates as cells grown in 2D. The P-gp encoding gene Mdr1a, however, was downregulated by $\sim 50 \%$ indicating significant changes in key renal transporters under shear stress. Changes in P-gp expression in cells exposed to shear stress has been reported in other studies as well. Jang et al. and Vriend et al. have reported an increase in P-gp expression in primary human proximal tubule epithelial cells using microfluidic cell culture systems [41, 42]. However, Jayagopal et al. have reported a decrease in P-gp expression in MDCK cells when shear stress was introduced [35].

Following the establishment of MDCK-Mdr1a cells' ability to grow under flow conditions, we have shown that Glucose and Lactate concentrations remain constant within the RM, compared to static Transwell cell cultures. This indicates the importance of a constant flow system compared to static cultures where the cycle of nutrient/metabolic waste accumulation/depletion causes significant changes to cell function [43].

The next step was to evaluate the MDCK-Mdr1a cells' ability to form an intact cell barrier. The formation of a tight cell monolayer in the HF surface is crucial for the model as this would allow the transfer of a compound through the renal cell active transport system rather than diffusion across a porous membrane. Due to the non-transparency of both the HF and RMs, an alternative approach was implemented. Media flowing through the HF lumen contained Inulin-FITC and the leakage to the ECS was measured daily. As cells cover the inner walls of the HF and form tight junctions, less Inulin-FITC is leaked and by day 10, almost none passes through indicating the presence of an intact cell barrier.

Active renal reabsorption and tubular secretion are crucial functions of the proximal tubule in the nephron, and it is initiated mainly by transporters located on the apical and basolateral sides of proximal tubule epithelial cells. Renal protein reabsorption is one of the key features of renal physiology where more than $60 \%$ of the absorption takes place at the apical membrane of cells in the proximal convoluted tubule [44]. Albumin is the most abundant protein in the plasma and it although a portion filters through the glomerulus, most is reabsorbed through receptor-mediated endocytosis in the proximal convoluted tubule and then into the bloodstream [45].

Unidirectional flux of fluorescently labelled BSA (BSAAF594) of cells grown in the RMs indicates that cells not only retain one of the most important functions of the proximal convoluted tubule, but they do so in greater efficiency than static Transwell cell cultures. The almost 30-fold increase in the $P_{\text {app }}$ ratio show large differences in albumin reabsorption between the two models.

Finally, we have studied P-gp function. As an ATPbinding cassette transporter located at apical membrane, it mediates the efflux of exogenous and endogenous compounds including potential therapeutics by secreting them into the tubular lumen [46, 47]. Hoechst and Rho123 are known P-gp-specific fluorescent substrates while Valspodar inhibits its action [48, 49]. Efflux assays have shown significant and minor differences in P-gp-mediated efflux between Transwell and RM cell cultures depending on the substrate. The reduction in substrate efflux $P_{\text {app }}$ ratios could be explained by several factors such the downregulation of P-gp expression described earlier or the slower diffusion of substrates across the HF. The Transwell membrane is $10 \mu \mathrm{m}$ thick, while the HF membrane is $150 \mu \mathrm{M}$ thick which could hinder substrate diffusion [50].

In conclusion, we have developed a custom-made HF-based bioreactor (RM) to be used for studying renal transport studies. Differences between Transwell and RM cell cultures were shown, making it a candidate as a 3D renal model. However, several factors could contribute to these changes other than shear stress and scaffold curvature. Oxygen concentration, cell interactions with the HF surface and pore size have not been properly addressed. In addition, the RM has not been compared to the active transport of renal cells in vivo to conclude if it induces cells grown in vitro to have a more physiological phenotype.

Further studies using pharmaceutical compounds and primary renal cells would be required to determine the possibility of using the RM as a drug-screening tool, while co-cultures with endothelial cells in the ECS could further enhance the imitation of the in vivo physiology.

Supplementary Information The online version contains supplementary material available at https://doi.org/10.1007/s10047-021-01260-w.

Acknowledgements We would like to thank Anthony Eyre and his workshop at Loughborough University for designing 3D models and manufacturing the renal modules. This work was funded by a joint BBSRC (Grant Code: BB/P50483X/1) and AstraZeneca CASE PhD studentship at Loughborough University.

\section{Declarations}

Conflict of interest The authors declare no conflict of interest.

Open Access This article is licensed under a Creative Commons Attribution 4.0 International License, which permits use, sharing, adaptation, distribution and reproduction in any medium or format, as long as you give appropriate credit to the original author(s) and the source, provide a link to the Creative Commons licence, and indicate if changes 
were made. The images or other third party material in this article are included in the article's Creative Commons licence, unless indicated otherwise in a credit line to the material. If material is not included in the article's Creative Commons licence and your intended use is not permitted by statutory regulation or exceeds the permitted use, you will need to obtain permission directly from the copyright holder. To view a copy of this licence, visit http://creativecommons.org/licenses/by/4.0/.

\section{References}

1. Scherer FM. R \& D costs and productivity in biopharmaceuticals. Regulatory policy program working paper RPP-2011-10; 2011.

2. DiMasi JA, Hansen RW, Grabowski HG. The price of innovation: new estimates of drug development costs. J Health Econ. 2003;22:151-85.

3. Nigam SK, Wu W, Bush KT, Hoenig MP, Blantz RC, Bhatnagar V. Handling of drugs, metabolites, and uremic toxins by kidney proximal tubule drug transporters. Clin J Am Soc Nephrol. 2015;10:2039-49.

4. Homan KA, et al. Bioprinting of 3D convoluted renal proximal tubules on perfusable chips. Sci Rep. 2016;6:34845.

5. Krajcsi P. Drug-transporter interaction testing in drug discovery and development. World J Pharmacol. 2013;2:35.

6. Colas C, Ung PM-U, Schlessinger A. SLC transporters: structure, function, and drug discovery. Med Chem Comm. 2016;7:1069-81.

7. Pelis RM, Wright SH. SLC22, SLC44, and SLC47 transporters-organic anion and cation transporters: molecular and cellular properties. Curr Top Membr. 2014;1:233-61.

8. Brajša $\mathrm{K}$, Trzun M, Zlatar I, Jelić D. Three-dimensional cell cultures as a new tool in drug discovery. Period Biol. 2016;118:59-65.

9. Ginai M, Elsby R, Hewitt CJ, Surry D, Fenner K, Coopman $\mathrm{K}$. The use of bioreactors as in vitro models in pharmaceutical research. Drug Discov Today. 2013;18:922-35.

10. Aebischer P, Ip TK, Panol G, Galletti PM. The bioartificial kidney: progress towards an ultrafiltration device with renal epithelial cells processing. Life Support Sys J Eur Soc Artif Organs. 1987;5:159-68.

11. Humes HD, MacKay SM, Funke AJ, Buffington DA. Tissue engineering of a bioartificial renal tubule assist device: in vitro transport and metabolic characteristics. Kidney Int. 1999;55:2502-14.

12. Humes HD, Buffington DA, MacKay SM, Funke AJ, Weitzel WF. Replacement of renal function in uremic animals with a tissue-engineered kidney. Nat Biotechnol. 1999;17:451-5.

13. Humes HD, et al. Initial clinical results of the bioartificial kidney containing human cells in ICU patients with acute renal failure. Kidney Int. 2004;66:1578-88.

14. Jansen J, Fedecostante M, Wilmer MJ, van den Heuvel LP, Hoenderop JG, Masereeuw R. Biotechnological challenges of bioartificial kidney engineering. Biotechnol Adv. 2014;32:1317-27.

15. Jansen J, et al. Human proximal tubule epithelial cells cultured on hollow fibers: living membranes that actively transport organic cations. Sci Rep. 2015;5:16702.

16. Schophuizen CMS, et al. Development of a living membrane comprising a functional human renal proximal tubule cell monolayer on polyethersulfone polymeric membrane. Acta Biomater. 2015;14:22-32.

17. Ni M, et al. Characterization of membrane materials and membrane coatings for bioreactor units of bioartificial kidneys. Biomaterials. 2011;32:1465-76.
18. Buschmann I, et al. The "Artificial Artery" as in vitro perfusion model. PLoS ONE. 2013;8:e57227.

19. Zhang H, Tasnim F, Ying JY, Zink D. The impact of extracellular matrix coatings on the performance of human renal cells applied in bioartificial kidneys. Biomaterials. 2009;30:2899-911.

20. Allen LT, et al. Surface-induced changes in protein adsorption and implications for cellular phenotypic responses to surface interaction. Biomaterials. 2006;27:3096-108.

21. Chevtchik NV, et al. Upscaling of a living membrane for bioartificial kidney device. Eur J Pharmacol. 2016;790:28-35.

22. Dumitrescu LN, et al. Induced hydrophilicity and in vitro preliminary and subsequent thermal treatment. Molecules. 2020;25:582.

23. Miner JH. Renal basement membrane components. Kidney Int. 1999;56:2016-24.

24. Yunoki S, Ohyabu Y, Hatayama H. Temperature-responsive gelation of type I collagen solutions involving fibril formation and genipin crosslinking as a potential injectable hydrogel. Int J Biomater. 2013;1:2013.

25. Doyle AD. Generation of 3D collagen gels with controlled, diverse architectures. Curr Protoc Cell Biol. 2017;72:1-22.

26. Nieskens TTG, et al. Nephrotoxicity and kidney transport assessment on 3D perfused proximal tubules. AAPS J. 2018;20:1-11.

27. King SM, Higgins JW, Nino CR, Smith TR, Paffenroth EH, Fairbairn CE, Docuyanan A, Shah VD, Chen AE, Presnell SC, Nguyen DG. 3D proximal tubule tissues recapitulate key aspects of renal physiology to enable nephrotoxicity testing. Front Physiol. 2017;8:123.

28. Duan Y, et al. Shear-induced reorganization of renal proximal tubule cell actin cytoskeleton and apical junctional complexes. Proc Natl Acad Sci. 2008;105:11418-23.

29. Bhatia SN, Ingber DE. Microfluidic organs-on-chips. Nat Biotech. 2014;32:760-72.

30. Kim S, et al. Pharmacokinetic profile that reduces nephrotoxicity of gentamicin in a perfused kidney-on-a-chip. Biofabrication. 2016;8:015021.

31. Thuenauer R, Rodriguez-Boulan E, Rümer W. Microfluidic approaches for epithelial cell layer culture and characterisation. Analyst. 2014;139:3206-18.

32. Sakolish CM, Mahler GJ. A novel microfluidic device to model the human proximal tubule and glomerulus. RSC Adv. 2017;7:4216-25.

33. Raghavan V, Rbaibi Y, Pastor-Soler NM, Carattino MD, Weisz OA. Shear stress-dependent regulation of apical endocytosis in renal proximal tubule cells mediated by primary cilia. Proc Natl Acad Sci USA. 2014;111:8506-11.

34. Vriend J, et al. Screening of drug-transporter interactions in a 3D microfluidic renal proximal tubule on a chip. AAPS J. 2018. https://doi.org/10.1208/s12248-018-0247-0.

35. Jayagopal A, et al. Apical shear stress enhanced organic cation transport in human OCT2/MATE1-transfected madin-darby canine kidney cells involves ciliary sensing. J Pharmacol Exp Ther. 2019;369:523-30.

36. Xie JX, Li X, Xie Z. Regulation of renal function and structure by the signaling Na/K-ATPase. IUBMB Life. 2013;65:991-8.

37. Corbeil D, et al. The human AC133 hematopoietic stem cell antigen is also expressed in epithelial cells and targeted to plasma membrane protrusions. J Biol Chem. 2000;275:5512-20.

38. Fargeas CA, Joester A, Missol-Kolka E, Hellwig A, Huttner WB, Corbeil D. Identification of novel prominin-1/CD133 splice variants with altrnative $\mathrm{C}$-termini and their expression in epididymis and testis. J Cell Sci. 2004;11:4301-11. 
39. Carbonic anhydrase 9 as an endogenous marker for hypoxic cells in cervical cancer-PubMed. Available: https://pubmed. ncbi.nlm.nih.gov/11751418/. Accessed 26 Jul 2020.

40. Bailly V, Zhang Z, Cate R, Sanicola M, Bonventre JV, Meier W. Shedding of kidney injury molecule-1, a putative adhesion protein involved in renal regeneration shedding of kidney injury molecule-1, a putative adhesion protein involved in renal regeneration. J Biol Chem. 2002;277:39739-48.

41. Vriend $\mathbf{J}$, et al. Flow stimulates drug transport in a human kidney proximal tubule-on-a-chip independent of primary cilia. Biochim Biophys Acta Gen Subj. 2020;1864:129433.

42. Jang K-J, et al. Human kidney proximal tubule-on-a-chip for drug transport and nephrotoxicity assessment. Integr Biol. 2013;5:1119.

43. Hughes P, Marshall D, Reid Y, Parkes H, Gelber C. Effects of over-subculturing. Biotechniques. 2007;43:575-86.

44. Birn H, Christensen EI. Renal albumin absorption in physiology and pathology. Kidney Int. 2006;69:440-9.

45. Ferrell N, Ricci KB, Groszek J, Marmerstein JT, Fissell WH. Albumin handling by renal tubular epithelial cells in a microfluidic bioreactor. Biotechnol Bioeng. 2012;109:797-803.

46. Zhang D, Luo G, Ding X, Lu C. Preclinical experimental models of drug metabolism and disposition in drug discovery and development. Acta Pharm Sin B. 2012;2:549-61.
47. Gubbins PO, Garimella TS, Dowling TC, Karyekar CS, Eddington ND. Evaluation of P-glycoprotein-mediated renal drug interactions in an MDR1-MDCK model. Pharmacotherapy. 2004;23:436-42.

48. Caetano-Pinto P, Janssen MJ, Gijzen L, Verscheijden L, Wilmer MJG, Masereeuw R. Fluorescence-based transport assays revisited in a human renal proximal tubule cell line. Mol Pharm. 2016;13:933-44.

49. Tang F, Ouyang H, Yang JZ, Borchardt RT. Bidirectional transport of rhodamine 123 and hoechst 33342, fluorescence probes of the binding sites on P-glycoprotein, across MDCK-MDR1 cell monolayers. J Pharm Sci. 2004;93:1185-94.

50. Ryan MJ, Johnson G, Kirk J, Fuerstenberg SM, Zager RA, TorokStorb B. HK-2: an immortalized proximal tubule epithelial cell line from normal adult human kidney. Kidney Int. 1994;45:48-57.

Publisher's Note Springer Nature remains neutral with regard to jurisdictional claims in published maps and institutional affiliations. 